Published in final edited form as:

Angew Chem Int Ed Engl. 2009 ; 48(2): 299-303. doi:10.1002/anie.200804851.

\title{
Hydrocyanines: A Class of Fluorescent Sensors That Can Image Reactive Oxygen Species in Cell Culture, Tissue, and In Vivo**
}

\section{Kousik Kundu,}

The Wallace H. Coulter Department of Biomedical Engineering and Parker H. Petit Institute of Bioengineering and Bioscience Georgia Institute of Technology, Atlanta, GA 30332 (USA)

\section{Sarah F. Knight,}

Cardiology Division, Department of Medicine Emory University School of Medicine, Atlanta, GA 30322 (USA)

\section{Nick Willett,}

Cardiology Division, Department of Medicine Emory University School of Medicine, Atlanta, GA 30322 (USA)

\section{Sungmun Lee,}

The Wallace H. Coulter Department of Biomedical Engineering and Parker H. Petit Institute of Bioengineering and Bioscience Georgia Institute of Technology, Atlanta, GA 30332 (USA)

Prof. W. Robert Taylor, and

The Wallace H. Coulter Department of Biomedical Engineering and Parker H. Petit Institute of Bioengineering and Bioscience Georgia Institute of Technology, Atlanta, GA 30332 (USA)

Cardiology Division, Department of Medicine Emory University School of Medicine, Atlanta, GA 30322 (USA)

Cardiology Division, Atlanta VA Medical Center, Decatur, GA 30032 (USA)

\section{Prof. Niren Murthy ${ }^{\star}$}

The Wallace H. Coulter Department of Biomedical Engineering and Parker H. Petit Institute of Bioengineering and Bioscience Georgia Institute of Technology, Atlanta, GA 30332 (USA)

\section{Keywords}

fluorescence; hydrocyanines; reactive oxygen species; superoxide

\begin{abstract}
The development of fluorescent probes for superoxide and the hydroxyl radical is a central problem in the field of chemical biology. ${ }^{[1]}$ Superoxide and the hydroxyl radical play a significant role in a variety of inflammatory diseases, and probes that can detect these reactive oxygen species (ROS) have tremendous potential as medical diagnostics and
\end{abstract}

\footnotetext{
** This work was supported by the Georgia Tech/Emory Center for the Engineering of Living Tissues (funded by NSF-EEC-9731643) (N.M.), NSF-BES-0546962 Career Award (N.M.), NIH UO1 HL80711-01 (N.M.), NIH R21 EB006418 (N.M.), and a J\&J/GT Health Care Innovation Seed Grant Proposal (N.M.).

*Fax: (+1) 404-894-4243, niren.murthy@ bme.gatech.edu.

Supporting information for this article is available on the WWW under http://dx.doi.org/10.1002/anie.200804851.
} 
research tools. ${ }^{[2-6]}$ Fluorescent sensors for superoxide and the hydroxyl radical, such as dihydroethidium (DHE), have been developed; however, they have had limited applicability because of their spontaneous autoxidation, rapid photobleaching, low emission wavelengths, and multiple reaction products with ROS. ${ }^{[7]} \mathrm{New}$ chemical probes for superoxide and the hydroxyl radical are therefore greatly needed. Herein, we present a new family of fluorescent ROS sensors, termed the hydrocyanines, which can be synthesized in one step from the commercially available cyanine dyes and can detect superoxide and the hydroxyl radical in living cells, tissue samples, and for the first time in vivo. We anticipate widespread interest in the hydrocyanines given their physical/chemical characteristics and ease of synthesis.

The synthesis of and mechanism by which hydrocyanines image ROS are shown in Figure 1a. The hydrocyanines are synthesized by reducing the iminium cations of the cyanine dyes with $\mathrm{NaBH}_{4}$. Hydrocyanines detect ROS through fluorescent imaging; they are weakly fluorescent because of their disrupted $\pi$ conjugation; however, oxidation with either superoxide or the hydroxyl radical dramatically increases their fluorescence by regenerating their extended $\pi$ conjugation. Figure $1 \mathrm{~b}$ demonstrates the proof of principle of this methodology. Hydro-IR-676 has minimal fluorescence; however, oxidation with superoxide causes a 100-fold increase in its fluorescence intensity. The cyanine dyes comprise a family of approximately 40 dyes,${ }^{[8-9]}$ and the reduction methodology presented in Figure 1a was used to synthesize several new fluorescent ROS sensors, which have the physical and chemical properties needed to detect intracellular and extracellular ROS, both in vitro and in vivo. For example, five new ROS sensors, termed hydro-Cy3, hydro-Cy5, hydro-Cy7, hydroIR-783, and hydro-ICG, were synthesized by reduction with $\mathrm{NaBH}_{4}$ (Table 1). These molecules have negligible fluorescence, as a result of reduction of their iminium cations; however, after reaction with either superoxide or the hydroxyl radical, they fluoresce at 560, $660,760,800$, and $830 \mathrm{~nm}$, respectively (see Table 1 and the Supporting Information for details). Hydro-Cy3, hydro-Cy5, hydro-IR-676, and hydro-Cy7 have the physical properties needed to detect intracellular ROS, in that they are initially membrane-permeable molecules; however, oxidation with intracellular ROS converts them into charged and membraneimpermeable molecules, which should accumulate within cells that are overproducing ROS. In contrast, hydro-ICG and hydro-IR-783 are charged and membrane-impermeable molecules and have the physical properties needed for measuring extracellular ROS production. Finally, several of the hydrocyanines, such as hydro-Cy7 and hydro-ICG, have near-infrared emission wavelengths and are therefore suitable for detecting ROS in vivo. Thus, $\mathrm{NaBH}_{4}$ reduction of the cyanine dyes represents a powerful synthetic methodology for generating fluorescent ROS sensors.

Cells produce several different types of reactive oxygen and nitrogen species. We therefore investigated the specificity of the hydrocyanines towards different reactive oxygen and nitrogen species. ${ }^{[10]}$ Figure 2 demonstrates that hydro-Cy 3 has high selectivity towards radical oxidants and superoxide and generates negligible fluorescence in the presence of $\mathrm{Fe}^{2+}$, singlet oxygen, hydrogen peroxide, nitric oxide, peroxynitrates, and various other oxidizing agents (hydro-Cy7 also has a similar specificity profile; see the Supporting Information for details). The selectivity of the hydrocyanines to ROS may arise from a reaction mechanism involving two consecutive one-electron oxidations, similar to DHE 
oxidation. Therefore, we anticipate that the hydrocyanines will provide information about the production of superoxide and other radical oxidants in cell culture and in vivo.

We investigated the ability of the hydrocyanines to quantify the hydroxyl radical ${ }^{[11]}$ in vitro, and its sensitivity was compared against that of DHE. Figure 3 demonstrates that both hydro-Cy3 and hydro-Cy7 can detect nanomolar levels of radical oxidants. They both had linear relationships $\left(I^{2}=0.99\right)$ between fluorescence intensity and hydrogen peroxide concentration (1-30 nм range) in the Fenton's reagent. This correlation was significantly better than that of DHE, which had an $r^{2}$ value of only 0.36 in this concentration range. The hydrocyanines also react rapidly with ROS; for an equimolar concentration of Fenton's reagent and hydro-Cy3 $(50 \mu \mathrm{M})$, the oxidation of hydro-Cy3 occurred with a half-life of 3 $\min$ (see the Supporting Information).

A major problem with DHE is its autoxidation in aqueous solutions, which creates high levels of background fluorescence and interferes with ROS measurements. The hydro cyanines should have higher stability against autoxidation than DHE, because of their lower kinetic and thermodynamic oxidation driving force, resulting from lower resonance stabilization of oxidation intermediates and products. We therefore measured the stability of the hydrocyanines to autoxidation. Figure 4 demonstrates that the hydrocyanines have higher stability to autoxidation than DHE. For example, hydro-Cy7 and hydro-Cy3 both had halflives of approximately 3 days in aqueous $\mathrm{pH} 7.4$ buffer at $37^{\circ} \mathrm{C}$ and were two orders of magnitude more stable than DHE, which had a halflife of only 30 min (Figure 4). The hydrocyanines therefore have the stability, sensitivity, and reactivity needed to detect ROS in biological samples.

We measured the ability of hydro-Cy3 to detect ROS production in rat aortic smooth muscle (RASM) cells, during angiotensin (Ang) II mediated cell signaling. ${ }^{[12]}$ Ang II mediated ROS production in aortic smooth muscle cells is implicated in the development of atherosclerosis and hypertension. ${ }^{[12]}$ There is therefore great interest in measuring ROS production from RASM cells stimulated with Ang II. Figure 5a-c demonstrates that hydro-Cy3 can detect ROS production in RASM cells during Ang II mediated cell signaling. For example, RASM cells incubated with Ang II and hydro-Cy3 displayed intense intracellular fluorescence (Figure 5b), whereas RASMs incubated with just hydro-Cy3 displayed significantly lower fluorescence (Figure 5a). Importantly, application of the superoxide dismutase mimetic TEMPOL resulted in a dramatic decrease in fluorescence from RASM cells treated with hydro-Cy3 and Ang II, demonstrating that the cellular fluorescence was due to intracellular ROS production (Figure 5c). Importantly, hydro-Cy3 caused no cellular toxicity under these experimental conditions $(100 \mu \mathrm{M})$ and also at higher concentrations $(1 \mathrm{~mm})$, as determined by the trypan blue cell viability assay (see the Supporting Information for details).

We also investigated the ability of hydro-Cy3 to image ROS production from live, explanted mouse aortas, after lipopolysaccharide (LPS) stimulation. The detection of ROS in live tissue provides information about ROS production in a physiologic environment that closely resembles in vivo conditions and therefore provides details about ROS production that cell culture cannot. C57B1/6 mice were treated with either $16 \mathrm{mg} \mathrm{kg}^{-1}$ lipopolysaccharide endotoxin (LPS) or phosphate-buffer saline (PBS) for $16 \mathrm{~h}$ and then euthanized. The aortas 
of these mice were then isolated and incubated with either hydro-Cy3 or hydro-Cy3 and TEMPOL for $15 \mathrm{~min}$. Sections of the aorta were made, fixed with $10 \%$ formalin, and then mounted en face. Figure $5 \mathrm{~d}-\mathrm{f}$ demonstrates that hydro-Cy3 can image ROS production in live tissue explants. Mice incubated with LPS and hydro-Cy3 displayed significantly higher fluorescence intensity than mice treated with just PBS and hydro-Cy3 (Figure 5e vs. d). For example, the total integrated fluorescence intensity of the LPS-treated slide (Figure 5e) was 183.2 versus 46.9 in the PBS-treated mice (Figure 5d); importantly, incubation with TEMPOL reduced the fluorescence of the LPS-treated slides to control levels of only 34.9 (Figure $5 \mathrm{f}$ ), demonstrating that the hydro-Cy3 fluorescence was due to ROS production.

A key benefit of the hydrocyanines is their high emission wavelengths, which makes them suitable for imaging ROS production in vivo. We investigated the ability of hydro-Cy7 to image ROS production in vivo generated by activated macrophages and neutrophils in an LPS model of acute inflammation. Nine C57B1/6 mice were divided into three groups. One group was given an intraperitoneal (i.p.) injection of LPS ( $1 \mathrm{mg}$ in $400 \mu \mathrm{L}$ saline), a second group was given saline $(400 \mu \mathrm{L})$, and a third control group was untreated. After $6 \mathrm{~h}$, the mice were anaesthetized, their abdominal fur was removed, and the LPS- and saline-treated mice were injected i.p. with hydro-Cy7 ( $5 \mathrm{nmol}$ in $50 \mu \mathrm{L}$ methanol). The mice were imaged as triplets, one from each group, by using a Kodak FX in vivo imager with a $700 \mathrm{~nm}$ excitation laser and a $790 \mathrm{~nm}$ emission filter. Figure 6a shows a representative fluorescent image of mice treated with LPS and hydro-Cy7 versus those treated with just saline and hydro-Cy7, and demonstrates that the LPS-treated mice had greater fluorescence intensity than salinetreated mice. The mean fluorescent intensity from the abdominal area from the three sets of mice was also quantified by using Image-Pro software (Figure 6b), and demonstrates that the LPS-treated mice had approximately twofold higher fluorescence intensity than the saline group. Taken together, these results demonstrate that hydro-Cy7 can image ROS production in vivo.

In summary, the hydrocyanines are a new family of ROS sensors, synthesized by $\mathrm{NaBH}_{4}$ reduction of commercially available cyanine dyes, which have excellent stability to autoxidation, tunable emission wavelengths, and nanomolar sensitivity to ROS. Furthermore, the hydrocyanines can image superoxide and the hydroxyl radical in cell culture, tissue explants, and for the first time in vivo. On the basis of these results, we anticipate that the hydrocyanines will have numerous applications as research tools and medical diagnostics.

\section{Experimental Section}

Representative procedure for the synthesis of hydrocyanines: Cy3 (100 mg, $0.2 \mathrm{mmol})$ was dissolved in methanol $(5 \mathrm{~mL})$, placed in a four-drum vial, and covered with aluminum foil. $\mathrm{NaBH}_{4}$ ( $3 \mathrm{mg}, 0.08 \mathrm{mmol}$ in $0.5 \mathrm{~mL}$ methanol) was added dropwise to the purple Cy3 solution, which was stirred for 10 min to generate a colorless solution. The reaction mixture was then stirred for an additional $10 \mathrm{~min}$, and the solvent was removed under reduced pressure. The resulting solid was dissolved in $10 \mathrm{~mL}$ dichloromethane and $5 \mathrm{~mL}$ water and vigorously shaken. The organic layer was extracted with additional dichloromethane $(5 \mathrm{~mL}$ $\times 2$ ), dried over anhydrous sodium sulfate, and the solvent was removed under reduced 
pressure. The reduced dye thus obtained was used without further purification. See the Supporting Information for detailed characterization of the products.

Detection of the hydroxyl radical (Figure 3): The hydroxyl radical was generated in situ by treating hydrogen peroxide with $\mathrm{Fe}^{2+}$ for the following experiments. To a solution $(1 \mathrm{~mL})$ of hydro-Cy3 in methanol (78 $\mu \mathrm{M})$, various quantities of a hydrogen peroxide stock solution were added to generate hydrogen peroxide concentrations in the range $2-30 \mathrm{~nm}$. Aqueous $\mathrm{Fe}^{2+}(1 \mu \mathrm{M})$ was then added to the hydro-Cy3/ $\mathrm{H}_{2} \mathrm{O}_{2}$ solution to generate a $200 \mathrm{~nm}$ concentration. The resulting solution was kept at ambient temperature for $5 \mathrm{~min}$, and the fluorescence intensity was measured $\left(\lambda_{\mathrm{ex}} / \lambda_{\mathrm{em}}=535 / 560 \mathrm{~nm}\right)$ against a reagent blank at the same time. The sensitivity of DHE $\left(\lambda_{\mathrm{ex}} / \lambda_{\mathrm{em}}=515 / 560 \mathrm{~nm}\right)$ and hydro-Cy $7\left(\lambda_{\mathrm{ex}} / \lambda_{\mathrm{em}}=\right.$ $735 / 760 \mathrm{~nm}$ ) towards the hydroxyl radical was measured under similar experimental conditions.

\section{Supplementary Material}

Refer to Web version on PubMed Central for supplementary material.

\section{References}

1. a) Mattson MP. Nature. 2004; 430:631-639. [PubMed: 15295589] b) Winterbourn CC. Nat. Chem. Biol. 2008; 4:278-286. [PubMed: 18421291]

2. a) Gao JJ, Xu KH, Tang B, Yin LL, Yang G, Wen A, Li G. FEBS J. 2007; 274:1725-1733. [PubMed: 17355258] b) Xu K, Liu X, Tang B, Yang G, An YL. Chem. Eur. J. 2007; 13:1411-1416. [PubMed: 17072931]

3. Koide Y, Urano Y, Kenmoku S, Kojima H, Nagano T. J. Am. Chem. Soc. 2007; 129:10324-10325. [PubMed: 17672465]

4. Maeda H, Yamamoto K, Kohno I, Hafsi L, Itoh N, Nakagawa S, Kanagawa N, Suzuki K, Uno T. Chem. Eur. J. 2007; 13:1946-1954. [PubMed: 17136791]

5. Robinson KM, Janes MS, Pehar M, Monette JS, Ross MF, Hagen TM, Murphy MP, Beckman JS. Proc. Natl. Acad. Sci. USA. 2006; 103:15038-15043. [PubMed: 17015830]

6. Shepherd J, Hilderbrand SA, Waterman P, Heinecke JW, Weissleder R, Libby P. Chem. Biol. 2007; 14:1221-1231. [PubMed: 18022561]

7. a) Zielonka J, Vasquez-Vivar J, Kalyanaraman B. Nat. Protoc. 2008; 3:8-21. [PubMed: 18193017] b) Zhao H, Joseph J, Fales HM, Sokoloski EA, Levine RL, Vasquez - Vivar J, Kalyanaraman B. Proc. Natl. Acad. Sci. USA. 2005; 102:5727-5732. [PubMed: 15824309]

8. Ntziachristos V, Schellenberger EA, Ripoll J, Yessayan D, Graves E, Bogdanov A, Josephson L, Weissleder R. Proc. Natl. Acad. Sci. USA. 2004; 101:12294. [PubMed: 15304657]

9. Choy G, Choyke P, Libutti SK. Mol. Imaging. 2003; 2:303-312. [PubMed: 14717329]

10. a) Miller EW, Tulyathan O, Isacoff EY, Chang CJ. Nat. Chem. Biol. 2007; 3:263-267. [PubMed: 17401379] b) Albers AE, Okreglak VS, Chang CJ. J. Am. Chem. Soc. 2006; 128:9640-9641. [PubMed: 16866512] c) Miller EW, Albers AE, Pralle A, Isacoff EY, Chang CJ. J. Am. Chem. Soc. 2005; 127:16652-16659. [PubMed: 16305254] d) Chang MCY, Pralle A, Isacoff EY, Chang CJ. J. Am. Chem. Soc. 2004; 126:15392-15393. [PubMed: 15563161] e) Srikun D, Miller EW, Domaille DW, Chang CJ. J. Am. Chem. Soc. 2008; 130:4596-4597. [PubMed: 18336027] f) Lee D, Khaja S, Velasquez CJ, Dasari M, Sun C, Petros J, Taylor WR, Murthy N. Nat. Mater. 2007; 6:765-769. [PubMed: 17704780]

11. Fenton's reagent has the possibility of generating radical species other than the hydroxyl radical, such as carbon-based radicals and other oxygen-based radicals; these species may also oxidize the hydrocyanines. 
12. Zafari AM, Ushio-Fukai M, Akers M, Yin Q, Shah A, Harrison DG, Taylor WR, Griendling KK. Hypertension. 1998; 32:488-490. [PubMed: 9740615] 


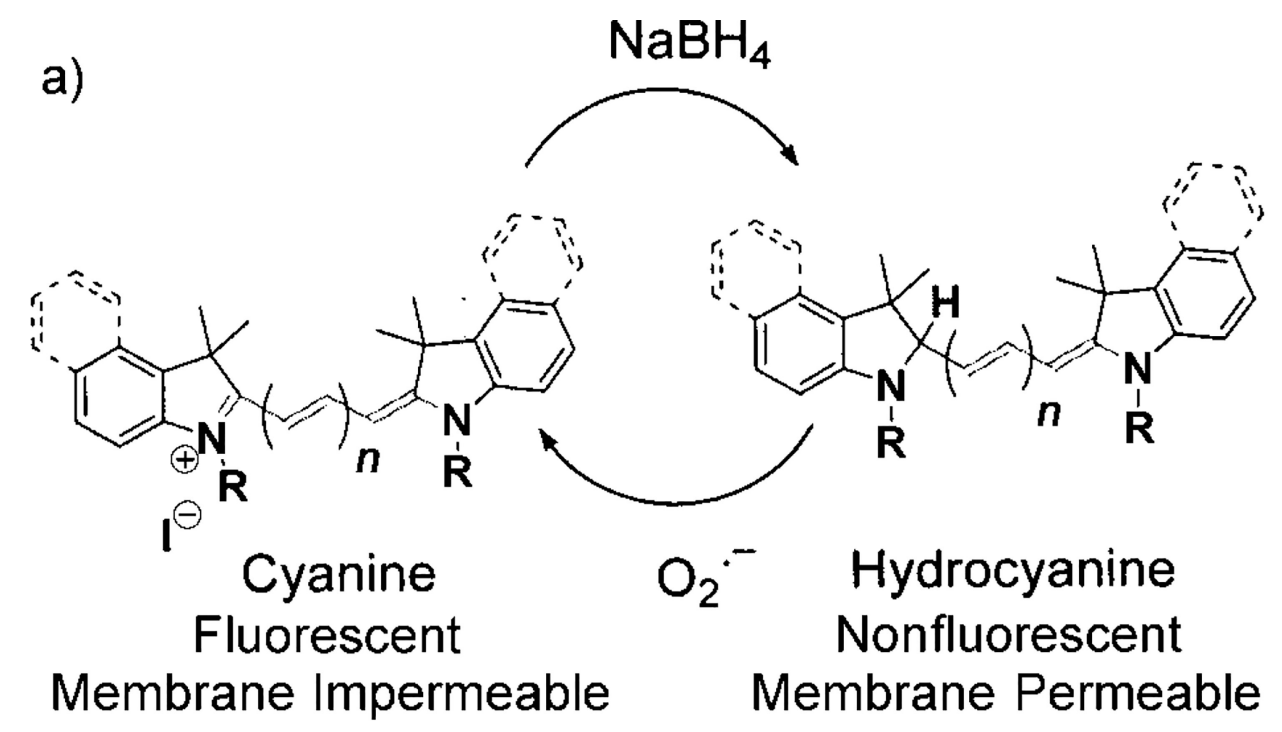

b)

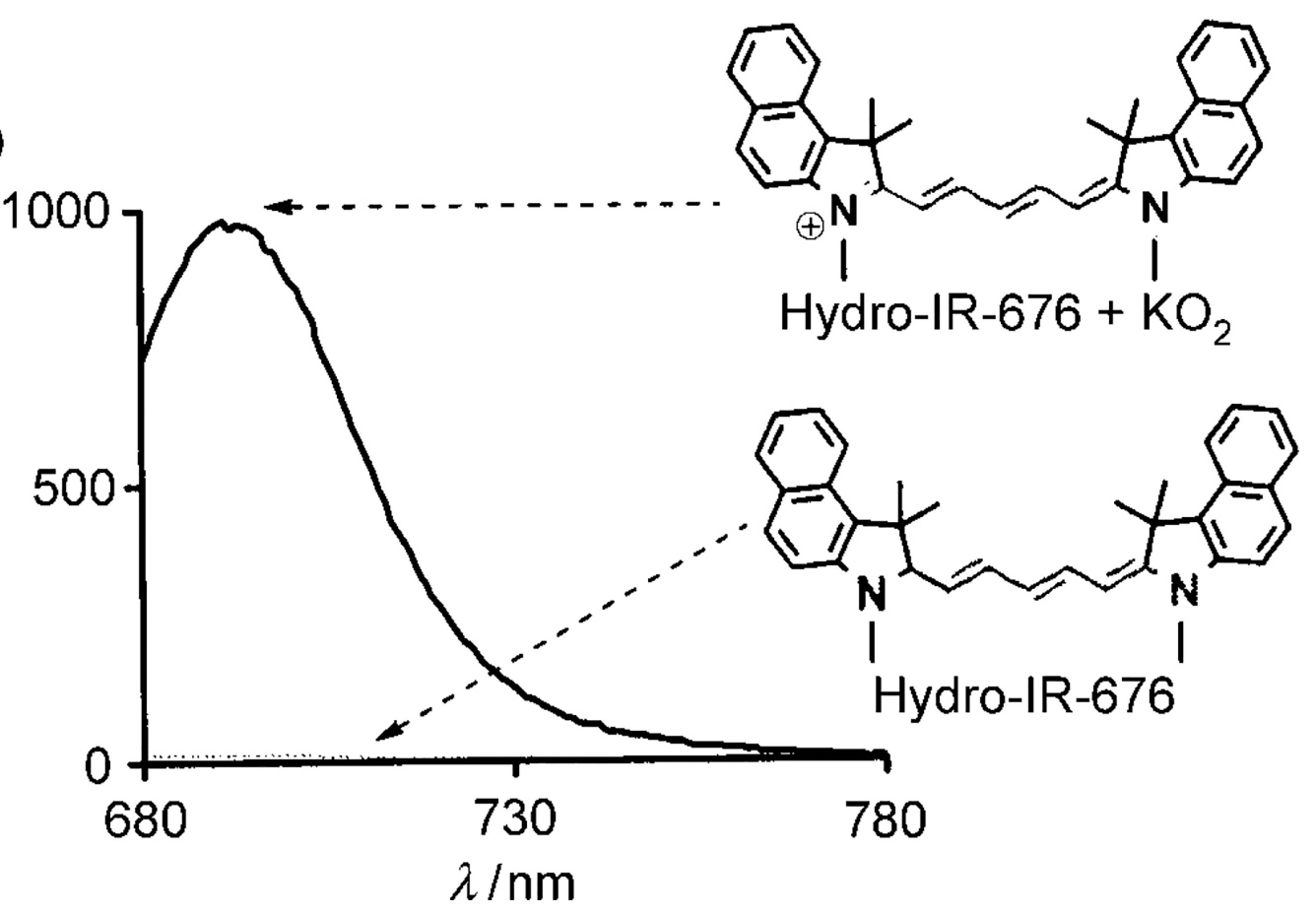

Figure 1.

a) Synthesis of hydrocyanines by a one-step reduction with $\mathrm{NaBH}_{4}$. Reaction with superoxide or the hydroxyl radical oxidizes the hydrocyanines to produce the fluorescent cyanine dyes. b) Hydro-IR-676 has negligible fluorescence emission; however, oxidation with superoxide causes a 100 -fold increase in fluorescence $\left(\lambda_{\mathrm{ex}}=675 \mathrm{~nm}, \lambda_{\mathrm{em}}=693 \mathrm{~nm}\right)$. 


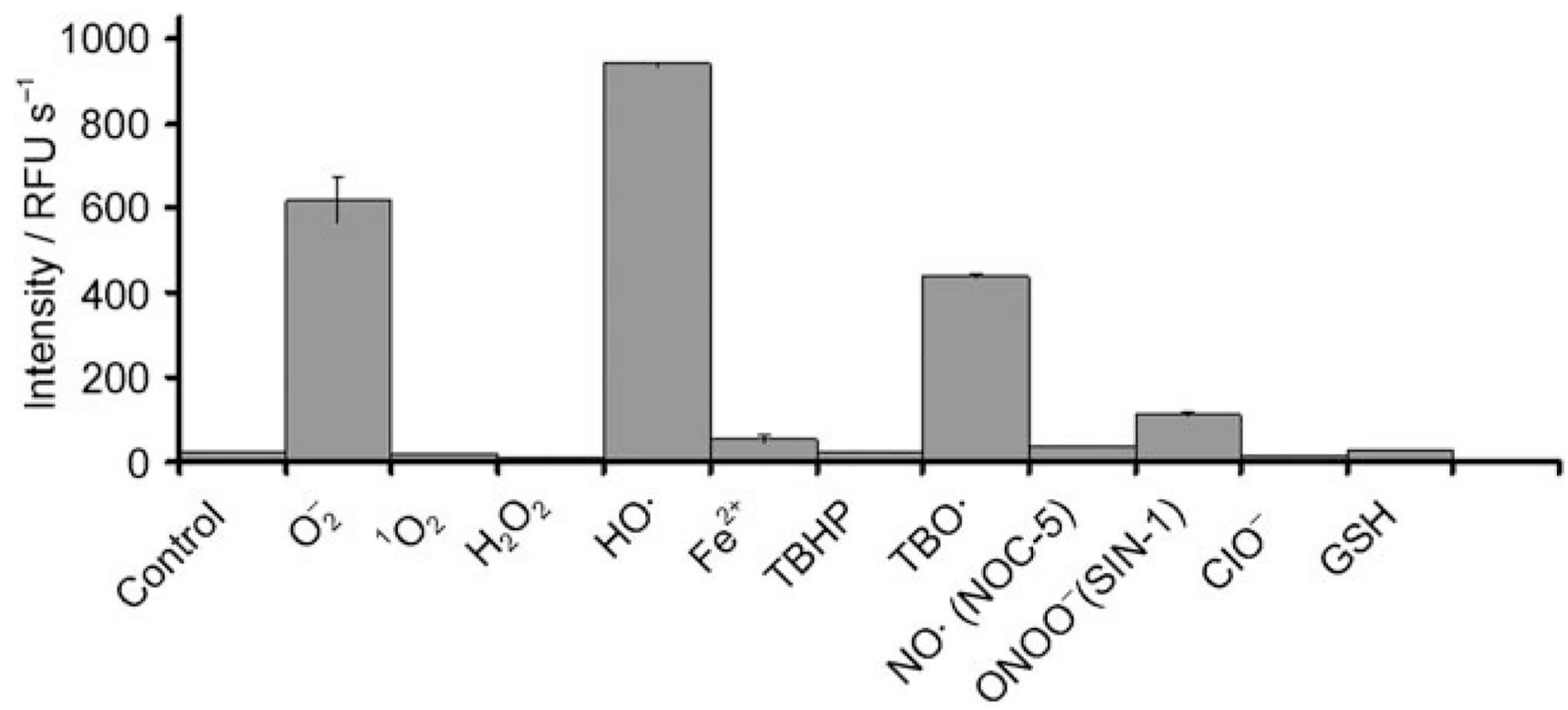

Figure 2.

Hydro-Cy3 has high specificity for superoxide and radical oxidants over other reactive oxygen and nitrogen species. (See the Supporting Information for details). RFU = relative fluorescence unit, TBHP = tert-butyl hydroperoxide, TBO $=$ tert-butyl peroxyl radical, NOC-5 = 3-(aminopropyl)-1-hydroxy-3-isopropyl-2-oxo-1-triazene, SIN-1 = 3-(4morpholinyl)sydnone imine hydrochloride, $\mathrm{GSH}=\boldsymbol{\gamma}$-L-glutamyl-L-cysteinyl-glycine . 


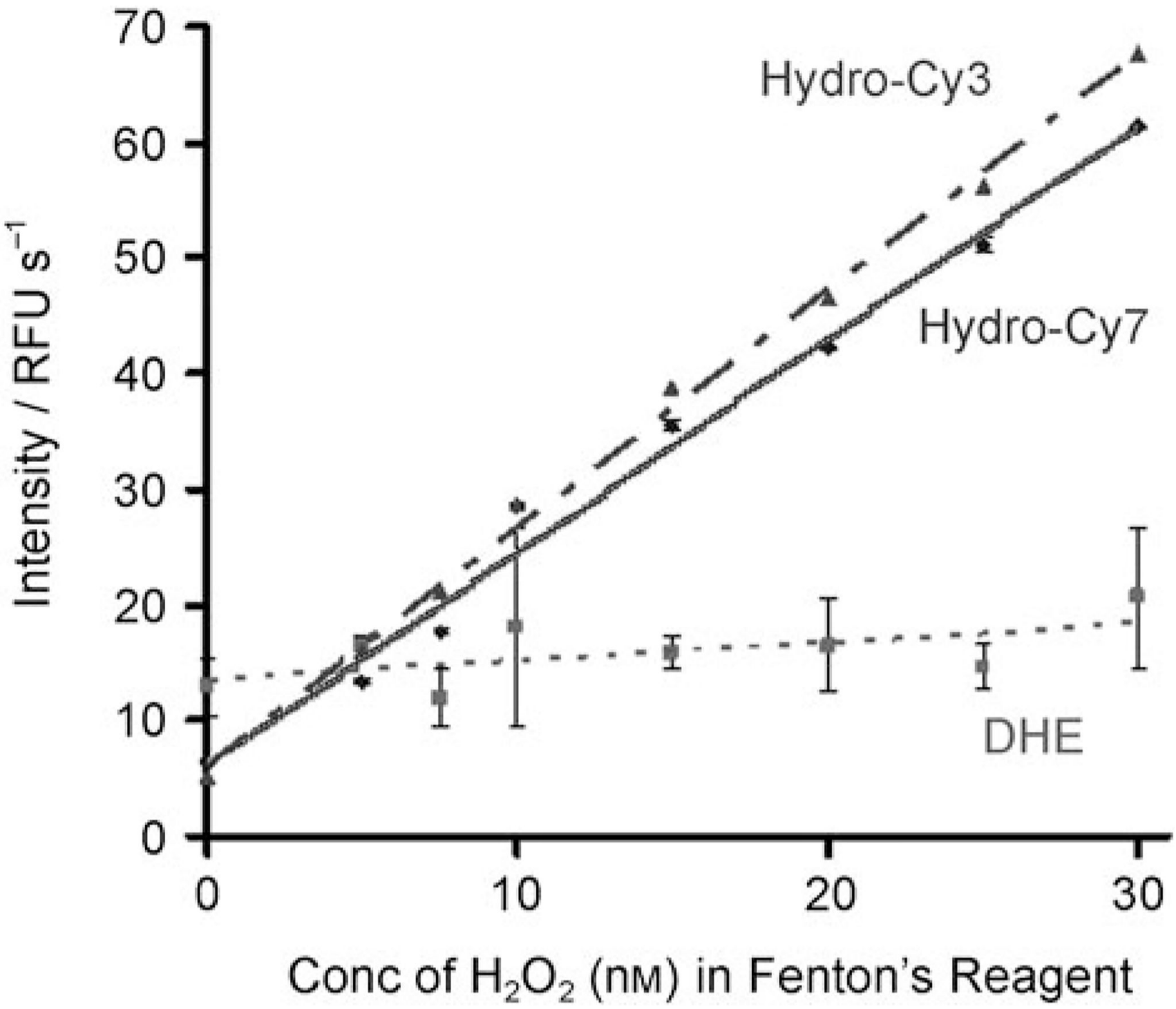

Figure 3.

Sensitivity of hydro-Cy3, hydro-Cy7, and DHE towards the hydroxyl radical. Both hydro$\mathrm{Cy} 3$ and hydro-Cy7 have nanomolar sensitivity towards the hydroxyl radical and are significantly more sensitive than DHE. 


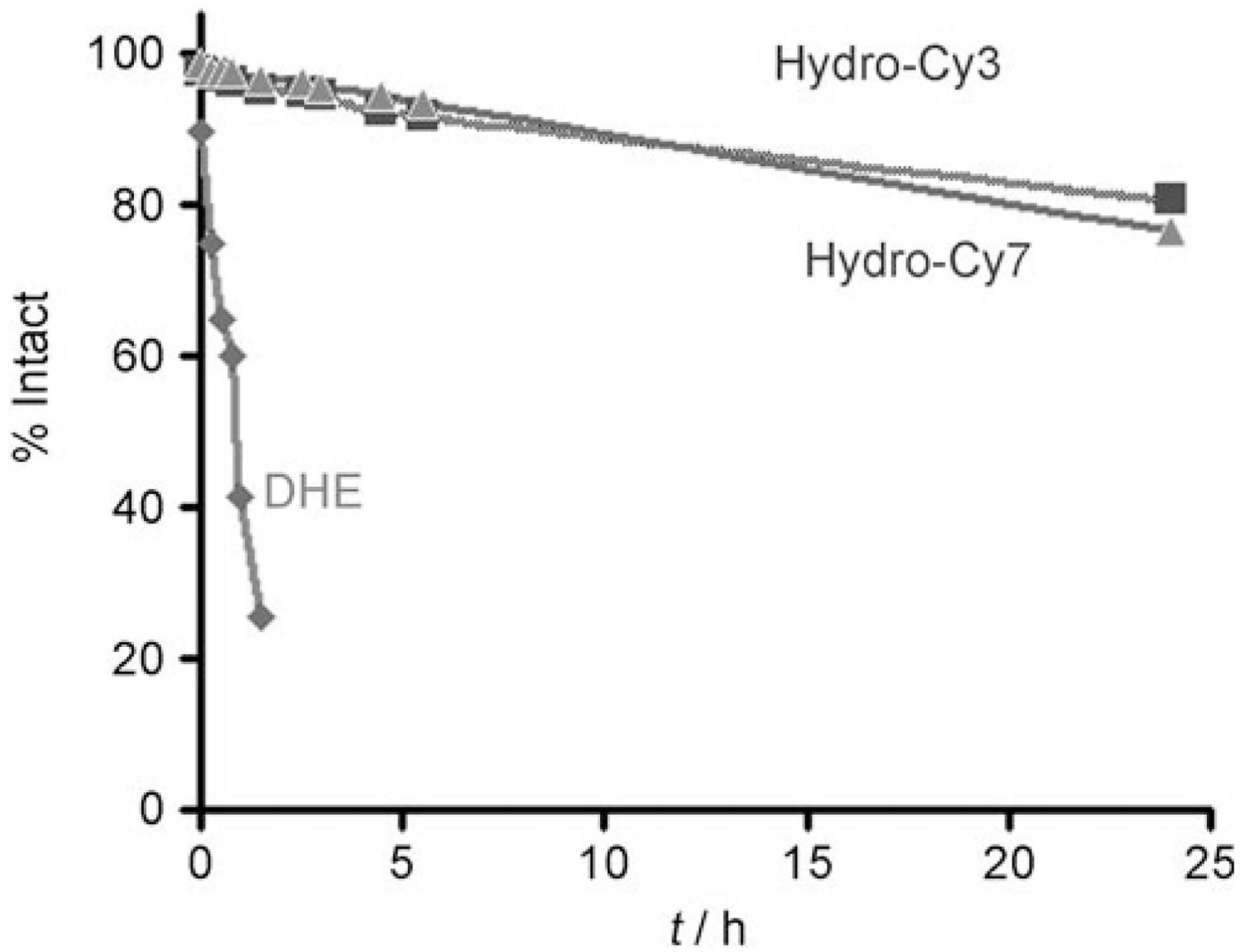

Figure 4.

Stability profile of hydro-Cy3, hydro-Cy7, and DHE towards autoxidation. The stability was measured in PBS buffer ( $\mathrm{pH} 7.4$ ) at $37^{\circ} \mathrm{C}$ (see the Supporting Information for details). 

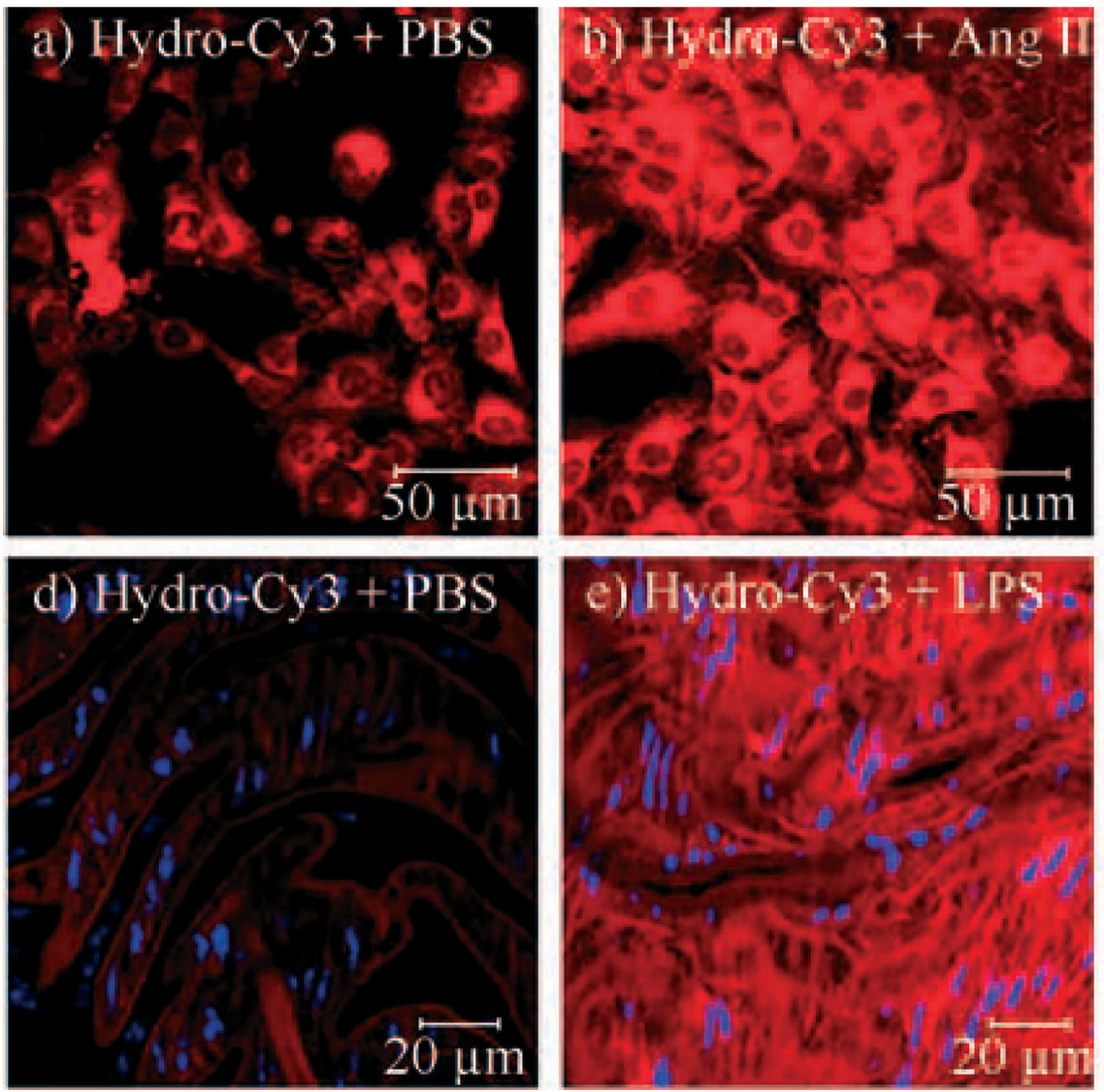

Figure 5.

Detection of ROS in live cells and tissue explants by hydrocyanines: a-c) confocal nuclei (blue dots).
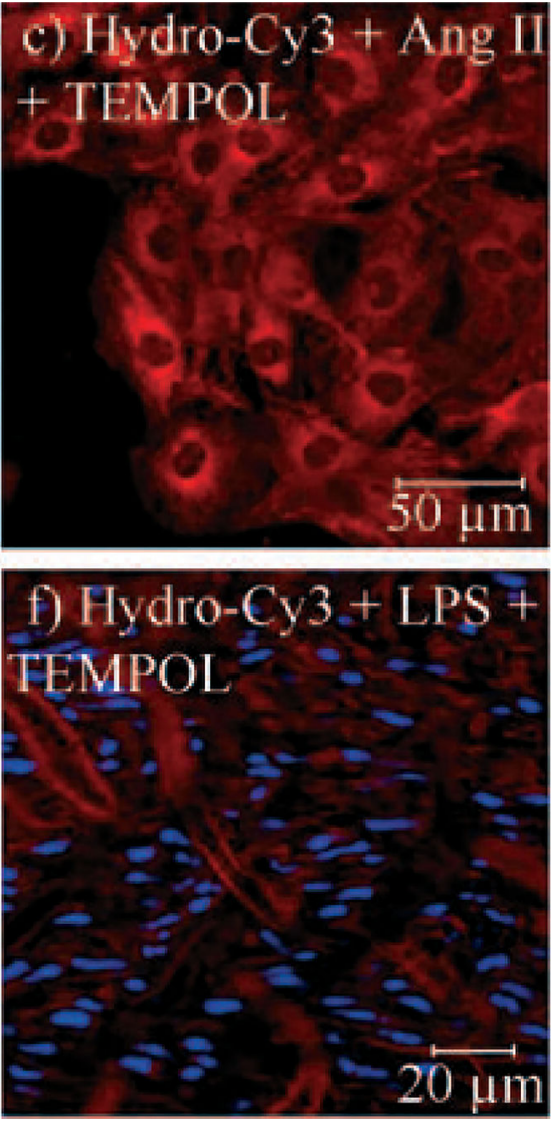
fluorescent images of live RASM cells: a) RASM cells incubated with $100 \mu$ м hydro-Cy3; b) RASM cells treated with Ang II (100 nM) for $4 \mathrm{~h}$ and incubated with $100 \mu \mathrm{M}$ hydro-Cy3; c) RASM cells incubated with Ang II (100 nM) for $4 \mathrm{~h}$ followed by $5 \mathrm{~mm}$ TEMPOL, prior to the addition of hydro-Cy3 $(100 \mu \mathrm{M}) ; \mathrm{d}-\mathrm{f})$ confocal fluorescent images of rat aortic tissue. Fluorescent image of the aorta section of d) the untreated mouse incubated with hydro-Cy3 for $15 \mathrm{~min}, \mathrm{e})$ the mouse treated with LPS incubated with hydro-Cy3 for $15 \mathrm{~min}$, and f) the mouse treated with LPS followed by incubation with $5 \mathrm{~mm}$ TEMPOL and hydro-Cy 3 for 15 min. Slides (d-f) were stained with $4^{\prime}, 6$-diamidino -2-phenylindole (DAPI) to identify cell 


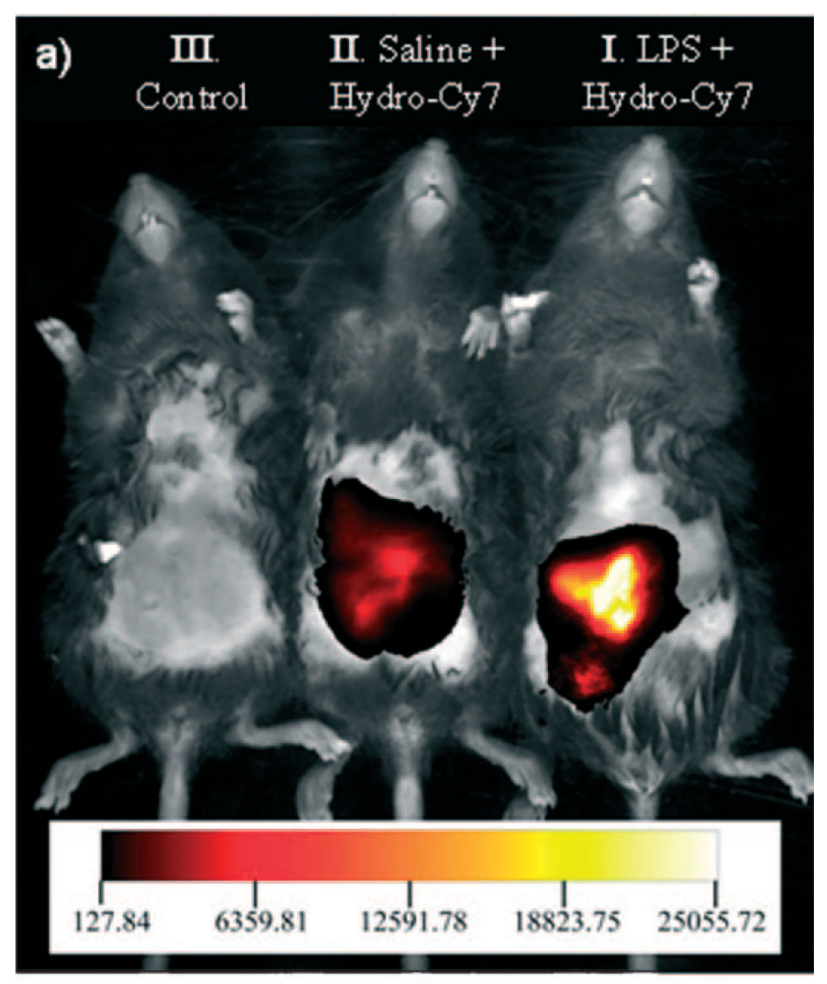

b) Quantification of in vivo fluorescence

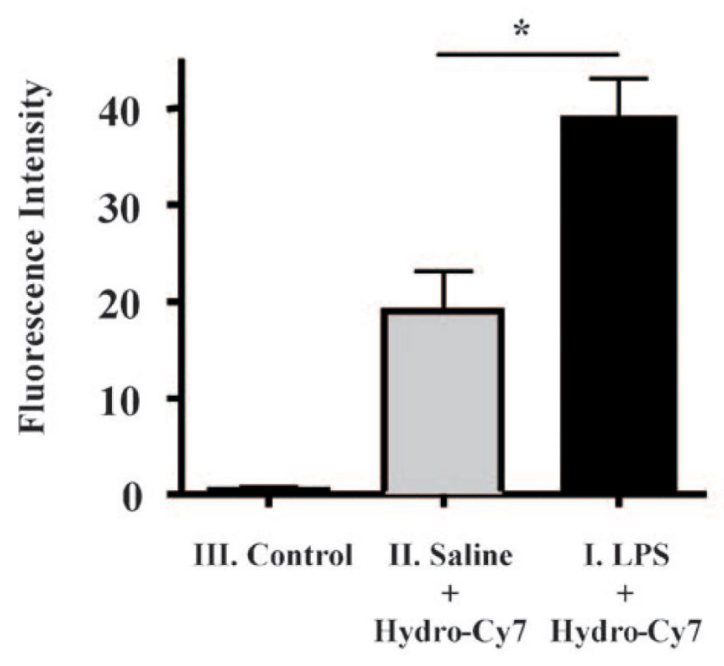

Figure 6.

In vivo imaging of ROS production from the peritoneal cavity of mice with hydro-Cy7 during an LPS-mediated inflammatory response: a) I) LPS was injected into the peritoneal cavity of the mice, followed by an i.p. injection of hydro-Cy7; II) saline was injected in the i.p. cavity of mice, followed by i.p. injection of hydro-Cy7; III) negative control, neither LPS nor hydro-Cy7 was injected. Mice from each group were imaged at the same time after hydro-Cy7 injection. b) Quantification of fluorescence intensities from LPS-treated mice, saline-treated mice, and a control (I, II, and III from (a), respectively). Oxidized hydro-Cy7 
fluorescence intensity is increased in LPS-treated mice relative to saline-treated mice ( $n=3$, 


\section{Table 1}

Hydrocyanines synthesized in high yields with tunable physical properties.

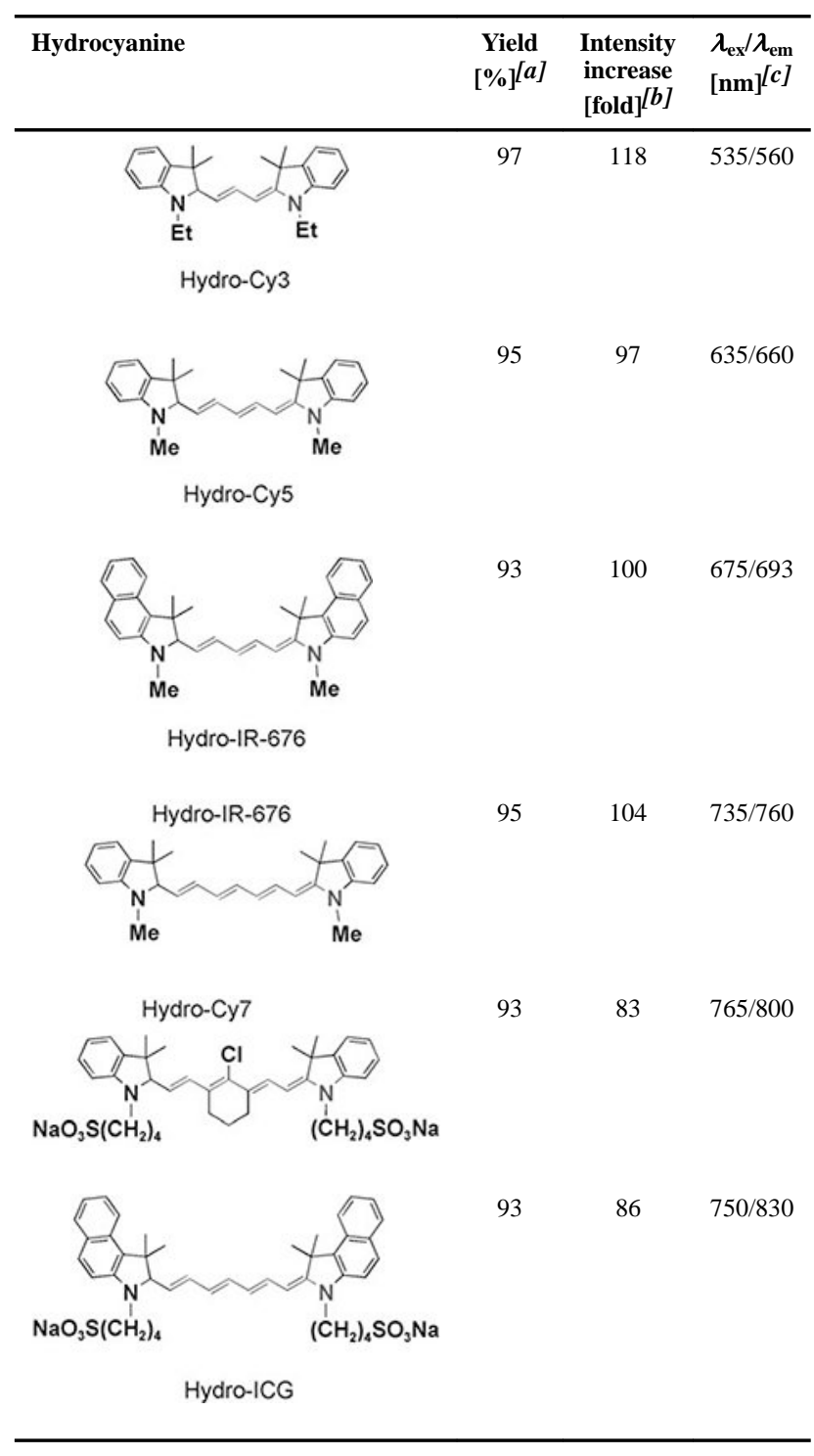

${ }^{[a]}$ Yields of isolated products.

${ }^{[b]}$ Fold increase was calculated by dividing the fluorescent intensity of oxidized hydrocyanine by the hydrocyanine fluorescence intensity. Concentrations of hydrocyanines and oxidized hydrocyanines were $7.5 \mu \mathrm{M}$ in $1 \mathrm{~mL}$ methanol. Hydrocyanines were oxidized with $20 \mathrm{mM} \mathrm{KO} 2$ in $20 \mu \mathrm{L}$ of DMSO.

${ }^{[c]}$ Excitation and emission wavelengths for the corresponding oxidized products. 